\title{
Anisotropic diffusion in confined colloidal dispersions: The evanescent diffusivity
}

\author{
James W. Swan a) and John F. Brady \\ Division of Chemistry and Chemical Engineering, California Institute of Technology, Pasadena, \\ California 91125, USA
}

(Received 13 November 2010; accepted 6 June 2011; published online 1 July 2011)

\begin{abstract}
We employ an analogy to traditional dynamic light scattering to describe the inhomogeneous and anisotropic diffusion of colloid particles near a solid boundary measured via evanescent wave dynamic light scattering. Following this approach, we generate new expressions for the short-time self- and collective diffusivities of colloidal dispersions with arbitrary volume fraction. We use these expressions in combination with accelerated Stokesian dynamics simulations to calculate the diffusivities in the limit of large and small scattering wave numbers for evanescent penetration depths ranging from four particle radii to one-fifth of a particle radius and volume fractions from $10 \%$ to $40 \%$. We show that at high volume fractions, and larger penetration depths, the boundaries have little effect on the dynamics of the suspension parallel to the wall since, to a first approximation, the boundary acts hydrodynamically much as another nearby particle. However, near and normal to the wall, the diffusivity shows a strong dependence on penetration depth for all volume fractions. This is due to the lubrication interactions between the particles and the boundary as the particle moves relative to the wall. These results are novel and comprehensive with respect to the range of penetration depth and volume fraction and provide a complete determination of the effect of hydrodynamic interactions on colloidal diffusion adjacent to a rigid boundary. () 2011 American Institute of Physics. [doi:10.1063/1.3604530]
\end{abstract}

\section{INTRODUCTION}

Dynamic light scattering (DLS) is one of the principle means of measuring the diffusivity of colloidal dispersions. ${ }^{1,2}$ In cases where particles are too small to observe via optical microscopy and diffuse too quickly to track the trajectory accurately, fluctuations in the density of the dispersion provide another means of directly observing the particle dynamics. In a light scattering experiment, one measures the time autocorrelation of the intensity of laser light scattered from the suspension. The zero-time, small scattering angle limit of the autocorrelation function is proportional to fluctuations in number density within the scattering volume, while the full time-dependent autocorrelation function is termed the intermediate scattering function. The time rate of change of this quantity is related to the diffusion of the scattering particles as the rate at which the intensity de-correlates is a measure of how fast the particles are moving.

This diffusivity is characterized by the time and length scales inherent to both the scattering experiment and the system under study. The scattering angle, related directly to the wavenumber of the scattered light (denoted $q$ ), sets the length scale over which density fluctuations are probed. Over short times and a length scale small relative to the particle size, itself denoted $a$, (this is the limit $q a \rightarrow \infty, t \rightarrow 0$ ), motion of a single particle is probed, and the short-time self-diffusivity is measured. Over short-time scales and large length scales indicative of small angle scattering $(q a \rightarrow 0, t \rightarrow 0)$, the

\footnotetext{
a) Author to whom correspondence should be addressed. Electronic mail: jswan@caltech.edu.
}

fluctuations in density are macro-scale and due to the collective motion of particles within the scattering volume. The measured dynamics are those characterized by the collective or gradient diffusivity which reflects the Fickian flux due to a macroscopic gradient in the suspension density. For long-times and over large length scales $(q a \rightarrow 0, t \rightarrow \infty)$, the long-time collective diffusivity is measured, whereas for small length scales $(q a \rightarrow \infty, t \rightarrow \infty)$ the long-time selfdiffusivity is measured. A comprehensive study of the relationship between fluctuations in the local density of the suspension and its short-time dynamics was performed by Rallison and Hinch. ${ }^{3}$ Similarly, Brady ${ }^{4}$ continued this line of investigation in pursuit of the long-time dynamics of the suspension. The key result for hard-sphere suspensions being that while the short-time correlation of the fluctuations in suspension density measure the short-time self-diffusivity, the long-time correlation is approximately the same rescaled by a factor related to the suspension microstructure, i.e.,

$$
D_{\infty}^{S}(\phi) \approx \frac{D_{0}^{S}(\phi)}{1+2 \phi g(2, \phi)}
$$

where $D_{\infty}^{S}(\phi)$ and $D_{0}^{S}(\phi)$ are the long- and short-time self-diffusivities, $\phi$ is the suspension volume fraction, and $\phi g(2, \phi)$ is a measure of the mean number of neighbors contacting a particular particle. Experiments employing this type of light scattering measure the hydrodynamic and structural properties isotropically and homogenously. Within a particular scattering volume, there is no mechanism to distinguish directionality or spatial variation in the hydrodynamic 
interactions or mean structure-two essential traits of colloidal dispersions in confined geometries.

Light scattered by evanescent waves, in contrast, samples a scattering volume heterogenously because the intensity of the evanescent wave (before it is scattered) decays exponentially fast with respect to distance from its origin. ${ }^{5}$ The decay rate is controlled by the so-called evanescent penetration depth, which parameterizes the measured light scattering intensity autocorrelation while distinguishing inhomogeneities in the suspension structure and hydrodynamic interactions. The illumination of the scattering volume is not uniform as with traditional light scattering but decays exponentially the deeper the volume penetrates the fluid.

It was Holmqvist et al. ${ }^{6}$ who showed experimentally that for very dilute suspensions (less that one-tenth of a percent volume fraction) the scattering due to evanescent waves also reveals the hydrodynamic anisotropy long anticipated theoretically. ${ }^{7-11}$ And indeed, we proposed a more general theory for the scattering of evanescent waves by Brownian particles in the short-time regime for suspensions of all volume fractions which exposes these same features. ${ }^{12}$ We used this general theory to predict the dynamics of dilute suspensions and confirmed the predictions by independent experimentation for volume fractions up to $1 \%$. Recent work has re-derived these same expressions and extended a virial expansion for the evanescent diffusivity to higher order. ${ }^{13}$ Further comparison between Stokesian Dynamics simulations and experiments has been made for volume fractions up to $42 \%,{ }^{14}$ though the complete details of the theory appear for the first time in the subsequent text. It was reserved until a comprehensive set of data for the short-time self- and collective diffusivities for a wide range of volume fractions and penetration depths was prepared.

The article proceeds in the following manner. In Sec. II, we derive expressions for the evanescent wave dynamic light scattering (EWDLS) diffusivities using an elementary approach derived from traditional light scattering analysis. In Sec. III, we describe the simulation methods implemented for modeling the hydrodynamic interactions among the particles using the Stokesian Dynamics method for suspensions bound within a channel. We also explain how both the static and dynamic measurements of the evanescent wave diffusivities may be made, though only the static approach is employed presently. In Secs. IV and V, we present the results of our simulations (the short-time self- and collective diffusivities) as a function of volume fraction and evanescent penetration depth and offer some physical interpretations of these results while concluding with a discussion of the applications for and experimental implications of this new technique.

\section{ANALYTICAL TECHNIQUES}

When a laser strikes the interface between a glass plane with refractive index $n_{1}$ and a solution with refractive index $n_{2}$ at a sufficiently high angle (measured with respect to the surface normal) such that it is totally reflected, an evanescent wave is formed on the solution side of the interface (see Fig. 1). This evanescent wave decays exponentially with

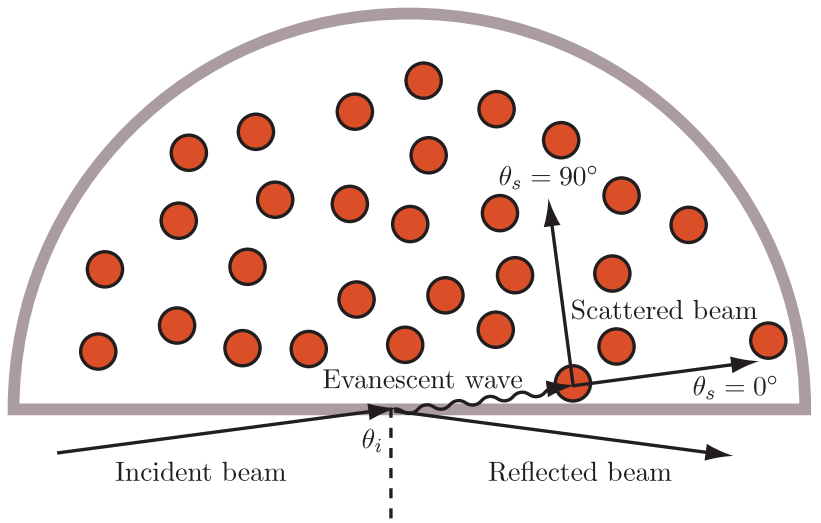

FIG. 1. An example of an EWDLS cell with the incident and scattering angles specifically labeled.

distance from the interface and scatters off the particles in the solution. The intensity of the scattered electric field due to a particle $\alpha$ is

$$
E_{\alpha}(\mathbf{q}, t ; \kappa)=I_{0} e^{-\frac{\kappa}{2} \mathbf{e}_{3} \cdot \mathbf{x}_{\alpha}^{t}+i \mathbf{q} \cdot \mathbf{x}_{\alpha}^{t}},
$$

where $I_{0}$ is the intensity of the unscattered field, $\kappa / 2$ is the inverse penetration depth of the evanescent wave governing the rate of exponential decay, $\mathbf{e}_{3}$ is the unit vector normal to the reflecting boundary, $\mathbf{x}_{\alpha}^{t}$ is the position of particle $\alpha$ relative to the wall at time $t$, and $\mathbf{q}$ is the difference between the incident and scattered beams. The penetration depth of the evanescent wave is a well-defined quantity that depends on the angle at which the incident beam strikes the interface, $\theta_{i}$, the vacuum wavelength of the laser, $\lambda_{0}$, and the refractive indices of the materials, viz.,

$$
\frac{\kappa}{2}=\frac{2 \pi}{\lambda_{0}} \sqrt{\left(n_{1} \sin \theta_{i}\right)^{2}-n_{2}^{2}} .
$$

Again, an evanescent wave is only emitted when the angle of incidence is greater than the critical angle, denoted $\theta_{c}$ and given by

$$
\theta_{i}>\theta_{c}=\arcsin \left(\frac{n_{1}}{n_{2}}\right) .
$$

One example of this process in a hemispherical experimental cell is illustrated in Fig. 1.

Because of the coherence of laser light, and in the limit that there is no multiple scattering, we can write the intensity of the beam scattered by many particles, $E(\mathbf{q}, t ; \kappa)$, as a linear superposition of that scattered by each individual particle,

$$
E(\mathbf{q}, t ; \kappa)=\sum_{\alpha=1}^{N} I_{0} e^{-\frac{\kappa}{2} \mathbf{e}_{3} \cdot \mathbf{x}_{\alpha}^{t}+i \mathbf{q} \cdot \mathbf{x}_{\alpha}^{t}}=\sum_{i=1}^{N} I_{0} e^{i \mathbf{k} \cdot \mathbf{x}_{\alpha}^{t}},
$$

where we have defined an effective, complex wave vector $\mathbf{k}$, such that

$$
\mathbf{k}=\mathbf{q}+i \frac{\kappa}{2} \mathbf{e}_{3}
$$

This effective wave vector will prove especially convenient in connecting the light scattering experiments to the dynamics of the suspension. It also corresponds to one particularly interesting interpretation of evanescent waves. Rather than having a real-valued wave vector, such as a typical 
electromagnetic wave, an evanescent wave has a complexvalued wave vector. This view is useful in that the conventional light scattering prescription ${ }^{1}$ for measuring particle dynamics can be followed with virtually no deviation. We derive the dynamics measured by evanescent waves in detail anyhow.

As in many light scattering experiments, the connection to the dynamics of the scattering medium is made through the time autocorrelation of the intensity of the scattered beam. The conjugate of the electric field intensity, $\bar{E}(\mathbf{q}, t ; \kappa)$, is simply

$$
\bar{E}(\mathbf{q}, t ; \kappa)=\sum_{i=1}^{N} I_{0} e^{-\frac{\kappa}{2} \mathbf{e}_{3} \cdot \mathbf{x}_{\alpha}^{t}-i \mathbf{q} \cdot \mathbf{x}_{\alpha}^{t}}=\sum_{\alpha=1}^{N} I_{0} e^{-i \overline{\mathbf{k}} \cdot \mathbf{x}_{\alpha}^{t}},
$$

and depends on the conjugate of the evanescent wave vector $(\overline{\mathbf{k}})$ instead of the wave vector itself as in conventional light scattering: $\overline{\mathbf{k}}=\mathbf{q}-i \kappa / 2 \mathbf{e}_{3}$.

We now concern ourselves with the correlation of the scattered electric field with its complex conjugate averaged over a large number of particle configurations or realizations. This is often called the intermediate scattering function and we denote it as $F(\mathbf{q}, t ; \kappa)$, viz.,

$$
\begin{aligned}
F(\mathbf{q}, t ; \kappa)= & \langle[E(\mathbf{q}, t ; \kappa)-\langle E(\mathbf{q}, t ; \kappa)\rangle] \\
& \times[\bar{E}(\mathbf{q}, 0 ; \kappa)-\langle\bar{E}(\mathbf{q}, 0 ; \kappa)\rangle]\rangle / I_{0}^{2} \\
= & \left\langle\sum_{\alpha, \beta=1}^{N} e^{i \mathbf{k} \cdot \mathbf{x}_{\beta}^{t}-i \overline{\mathbf{k}} \cdot \mathbf{x}_{\alpha}^{0}}\right\rangle-\left\langle\sum_{\alpha=1}^{N} e^{i \mathbf{k} \cdot \mathbf{x}_{\alpha}^{t}}\right\rangle\left\langle\sum_{\beta=1}^{N} e^{-i \overline{\mathbf{k}} \cdot \mathbf{x}_{\beta}^{0}}\right\rangle .
\end{aligned}
$$

The angular brackets denote an average over a large number of configurations of scattering particles in solution. This may be represented mathematically as an integral over the quantity in the brackets multiplied by the probability of finding the particles in that configuration. We make one additional simplification by noting that the particles in the solution are not tagged and may be thought of as identical. With this, we write the intermediate scattering function as

$$
\begin{aligned}
F(\mathbf{q}, t ; \kappa)= & \frac{1}{(N-1) !} \int\left[e^{i \mathbf{k} \cdot \mathbf{x}_{1}^{t}}+(N-1) e^{i \mathbf{k} \cdot \mathbf{x}_{2}^{t}}\right] \\
& \times e^{-i \overline{\mathbf{k}} \cdot \mathbf{x}_{1}^{0}} P_{N}^{t, 0} d \mathbf{x}^{N, t} d \mathbf{x}^{N, 0} \\
& -\delta\left[\left(\mathbf{I}-\mathbf{e}_{3} \mathbf{e}_{3}\right) \cdot \mathbf{k}\right] G\left(q_{\perp}, t ; \kappa\right) \bar{G}\left(q_{\perp}, 0 ; \kappa\right),
\end{aligned}
$$

where $P_{N}^{t, 0}$ is the joint probability of finding all $N$ particles in the configuration denoted $\mathbf{x}^{N, t}$ at time $t$ and in configuration denoted $\mathbf{x}^{N, 0}$ initially and $q_{\perp}=\mathbf{e}_{3} \cdot \mathbf{q}$. The factor proportional to $\delta\left[\left(\mathbf{I}-\mathbf{e}_{3} \mathbf{e}_{3}\right) \cdot \mathbf{k}\right]$, a delta function, is the square of

$$
G\left(q_{\perp}, t ; \kappa\right)=\int e^{i q_{\perp} z-\kappa z} n(z, t) d z,
$$

where $n(z, t)$ is the density distribution of the suspension as a function of the coordinate normal to the wall $(z)$. We assume for the remainder of the article that the process being probed is stationary so that $n(z, t)$ is constant in time. The same factor, $\left|G\left(q_{\perp}, t ; \kappa\right)\right|^{2} \delta\left[\left(\mathbf{I}-\mathbf{e}_{3} \mathbf{e}_{3}\right) \cdot \mathbf{k}\right]$, arises in conventional light scattering theory as $\langle N\rangle^{2} \delta(\mathbf{q})$ and is here generalized to EWDLS. We will investigate the peculiarities of its form as well as its significance after developing the full theory. For convenience, we define a new probability distribution $\hat{P}_{N}^{t}$, such that

$$
\hat{P}_{N}^{t}=\int P_{N}^{t \mid 0} e^{-i \overline{\mathbf{k}} \cdot \mathbf{x}_{1}^{0}} P_{N}^{0} d \mathbf{x}^{N, 0}
$$

where $P_{N}^{t \mid 0}$ is the conditional probability of finding the particles in configuration $\mathbf{x}^{N, t}$ at time $t$ given they began in configuration $\mathbf{x}^{N, 0}$, and $P_{N}^{0}$ is the probability of finding the particles in configuration $\mathbf{x}^{N, 0}$ initially. Using this, we rewrite the intermediate scattering function as

$$
\begin{aligned}
F(\mathbf{q}, t ; \kappa)= & \frac{1}{(N-1) !} \int\left[e^{i \mathbf{k} \cdot \mathbf{x}_{1}^{t}}+(N-1) e^{i \mathbf{k} \cdot \mathbf{x}_{2}^{t}}\right] \hat{P}_{N}^{t} d \mathbf{x}^{N, t} \\
& -\delta\left[\left(\mathbf{I}-\mathbf{e}_{3} \mathbf{e}_{3}\right) \cdot \mathbf{k}\right] G\left(q_{\perp}, t ; \kappa\right) \bar{G}\left(q_{\perp}, 0 ; \kappa\right) .
\end{aligned}
$$

Consider first the contribution to the intermediate scattering function due only to the correlation of scattering from a single particle, called the self-intermediate scattering function

$$
\begin{aligned}
F_{S}(\mathbf{q}, t ; \kappa)= & \frac{1}{(N-1) !} \int e^{i \mathbf{k} \cdot \mathbf{x}_{1}^{t}} \hat{P}_{N}^{t} d \mathbf{x}^{N, t} \\
& -\delta\left[\left(\mathbf{I}-\mathbf{e}_{3} \mathbf{e}_{3}\right) \cdot \mathbf{k}\right] G\left(q_{\perp}, t ; \kappa\right) \bar{G}\left(q_{\perp}, 0 ; \kappa\right) .
\end{aligned}
$$

The intermediate scattering function then can be written as

$$
F(\mathbf{q}, t ; \kappa)=F_{S}(\mathbf{q}, t ; \kappa)+\frac{1}{(N-2) !} \int e^{i \mathbf{k} \cdot \mathbf{x}_{2}^{t}} \hat{P}_{N}^{t} d \mathbf{x}^{N, t}
$$

In the limit that $t \rightarrow 0$, the probability density $\hat{P}_{N}^{t}$ becomes

$$
\hat{P}_{N}^{t}=\int \delta\left(\mathbf{x}^{N, t}-\mathbf{x}^{N, 0}\right) e^{-i \overline{\mathbf{k}} \cdot \mathbf{x}_{1}^{0}} P_{N}^{0} d \mathbf{x}^{N, 0},
$$

where we have made the substitution $P_{N}^{t \mid 0}=\delta\left(\mathbf{x}^{N, t}-\mathbf{x}^{N, 0}\right)$ as $t$ approaches zero. Therefore, we write the self-intermediate scattering function at $t=0$ as

$$
\begin{aligned}
F_{S}(\mathbf{q}, 0 ; \kappa)= & \frac{1}{(N-1) !} \int e^{-\kappa \mathbf{e}_{3} \cdot \mathbf{x}_{1}^{0}} P_{N}^{0} d \mathbf{x}^{N, 0} \\
& -\delta\left[\left(\mathbf{I}-\mathbf{e}_{3} \mathbf{e}_{3}\right) \cdot \mathbf{k}\right] G\left(q_{\perp}, 0 ; \kappa\right) \bar{G}\left(q_{\perp}, 0 ; \kappa\right),
\end{aligned}
$$

and the intermediate scattering function at $t=0$ as

$$
\begin{aligned}
F(\mathbf{q}, 0 ; \kappa)= & F_{S}(\mathbf{q}, 0 ; \kappa) \\
& +\frac{1}{(N-2) !} \int e^{i \mathbf{k} \cdot\left(\mathbf{x}_{2}^{0}-\mathbf{x}_{1}^{0}\right)-\kappa \mathbf{e}_{3} \cdot \mathbf{x}_{1}^{0}} P_{N}^{0} d \mathbf{x}^{N, 0} .
\end{aligned}
$$

Recognize that the integrand of the zero-time selfintermediate scattering function is a probability density weighted by the exponential decay of the evanescent wave. We can see this more clearly by rewriting it as

$$
\begin{aligned}
F_{S}(\mathbf{q}, 0 ; \kappa)= & \int e^{-\kappa \mathbf{e}_{3} \cdot \mathbf{x}_{1}^{0}} P_{1}^{0} d \mathbf{x}_{1}^{0} \\
& -\delta\left[\left(\mathbf{I}-\mathbf{e}_{3} \mathbf{e}_{3}\right) \cdot \mathbf{k}\right] G\left(q_{\perp}, 0 ; \kappa\right) \bar{G}\left(q_{\perp}, 0 ; \kappa\right),
\end{aligned}
$$

where $P_{1}^{0}$ is the probability of finding a particle at location $\mathbf{x}_{1}^{0}$ initially. This is calculated by averaging the initial probability density over the positions of the other $N-1$ particles, viz.,

$$
P_{1}^{0}=\frac{1}{(N-1) !} \int P_{N}^{0} d \mathbf{x}^{N-1,0} .
$$


Similarly, the zero-time intermediate scattering function is

$$
F(\mathbf{q}, 0 ; \kappa)=F_{S}(\mathbf{q}, 0 ; \kappa)+\int e^{i \mathbf{k} \cdot\left(\mathbf{x}_{2}^{0}-\mathbf{x}_{1}^{0}\right)-\kappa \mathbf{e}_{3} \cdot \mathbf{x}_{1}^{0}} P_{2}^{0} d \mathbf{x}_{1}^{0} d \mathbf{x}_{2}^{0},
$$

where $P_{2}^{0}$ is the probability of finding a pair of particles at positions $\mathbf{x}_{1}^{0}$ and $\mathbf{x}_{2}^{0}$ initially such that

$$
P_{2}^{0}=\frac{1}{(N-2) !} \int P_{N}^{0} d \mathbf{x}^{N-2,0} .
$$

Were this conventional light scattering $(\kappa \rightarrow 0)$, we would recognize the quantity $F(\mathbf{q}, 0 ; 0)$ as the static structure factor $(S(\mathbf{q}))$; however, because this is the correlation of scattering by an evanescent wave, the integrand of the static structure factor is modulated by an exponential decay. These static quantities are measurements of the configuration of the scattering particles only and contain no information about their dynamic behavior. Here, we redesignate the zero-time intermediate scattering function as the evanescent static structure factor and denote it $S(\mathbf{q}, \kappa)$.

Consider now, the time derivative of the self-intermediate scattering function

$$
\frac{\partial}{\partial t} F_{S}(\mathbf{q}, t ; \kappa)=\frac{1}{(N-1) !} \int e^{i \mathbf{k} \cdot \mathbf{x}_{1}^{t}} \frac{\partial \hat{P}_{N}^{t}}{\partial t} d \mathbf{x}^{N, t} .
$$

To describe this quantity further, we require information about how the conditional probability density changes as a function of time. This can be obtained from the $N$-particle Smoluchowski equation

$$
\frac{\partial P_{N}^{t \mid 0}}{\partial t}+\sum_{\alpha=1}^{N} \nabla_{\mathbf{x}_{\alpha}^{t}} \cdot \mathbf{j}_{\alpha}=0,
$$

where $\mathbf{j}_{\alpha}$ is the flux of probability density associated with the inter-particle and thermal forces on particle $\alpha$ given by

$$
\mathbf{j}_{\alpha}=\sum_{\beta=1}^{N} \mathbf{M}_{\alpha \beta} \cdot\left(\mathbf{F}_{\beta}^{P}-k T \nabla_{\mathbf{x}_{\beta}^{t}} \log P_{N}^{t \mid 0}\right) P_{N}^{t \mid 0} .
$$

Here, $\mathbf{M}_{\alpha \beta}$ is the hydrodynamic mobility tensor coupling forces on particle $\beta$ to the velocity of particle $\alpha$ in the presence of the wall, $\mathbf{F}_{\beta}^{P}$ is the inter-particle force on particle $\beta$ due to non-hydrodynamic interactions with other particles and with the wall, and $k T$ is the thermal energy (an external force or a shearing motion could also be added to $\mathbf{F}_{\beta}^{P}$ ). The mobility tensor is a purely geometric quantity that describes the motion of the particles in the fluid domain bounded by the wall. The additional resistance and hydrodynamic screening associated with particle motion in bounded geometries is included explicitly in these terms. Multiplying these expressions by $\exp \left(-i \overline{\mathbf{k}} \cdot \mathbf{x}_{1}^{0}\right) P_{N}^{0}$ and integrating over the initial configuration allows us to write a differential equation for the modified probability density

$$
\frac{\partial \hat{P}_{N}^{t}}{\partial t}=-\sum_{\alpha=1}^{N} \nabla_{\mathbf{x}_{\alpha}^{t}} \cdot \hat{\mathbf{j}}_{\alpha},
$$

where $\hat{\mathbf{j}}_{\alpha}$ is defined by analogy with $\hat{P}_{N}^{t}$ (see Eq. (15)). Substituting this into Eq. (22), integrating by parts and noting that the flux of particles decays to zero far away (and at particleparticle or particle-wall contact) yields

$$
\frac{\partial}{\partial t} F_{S}(\mathbf{q}, t ; \kappa)=\frac{1}{(N-1) !} \int e^{i \mathbf{k} \cdot \mathbf{x}_{i}^{t}} i \mathbf{k} \cdot \hat{\mathbf{j}}_{1} d \mathbf{x}^{N, t} .
$$

A similar set of manipulations allows us to write the time derivative of the intermediate scattering function as

$$
\frac{\partial}{\partial t} F(\mathbf{q}, t ; \kappa)=\frac{\partial}{\partial t} F_{S}(\mathbf{q}, t ; \kappa)+\frac{1}{(N-2) !} \int e^{i \mathbf{k} \cdot \mathbf{x}_{2}^{t}} i \mathbf{k} \cdot \hat{\mathbf{j}}_{2} d \mathbf{x}^{N, t}
$$

If we assert that the suspension was initially in equilibrium such that

$$
P_{N}^{0} \sim P_{N}^{e q} \sim e^{-V / k T},
$$

where $P_{N}^{e q}$ is the equilibrium probability density (adjacent to the wall) and $\mathbf{F}_{\beta}^{P}=-\nabla_{\mathbf{x}_{\beta}} V$, then the zero-time fluxes are

$$
\left.\hat{\mathbf{j}}_{1}\right|_{t=0}=\left.i k T \mathbf{M}_{11} \cdot \overline{\mathbf{k}} \hat{P}_{N}^{t}\right|_{t=0}
$$

and

$$
\left.\hat{\mathbf{j}}_{2}\right|_{t=0}=\left.i k T \mathbf{M}_{21} \cdot \overline{\mathbf{k}} \hat{P}_{N}^{t}\right|_{t=0} .
$$

Implicit in the definition of the inter-particle force above is the fact that a shearing motion cannot be admitted. There are approaches to including this physical behavior in the light scattering construction though we do not take them up here. ${ }^{19,20}$ Therefore, by writing the usual zero-time scattering function operation-the time derivative of the logarithm of the scattering autocorrelation-we find that for the self-intermediate scattering function

$$
\frac{\partial}{\partial t} \log F_{S}(\mathbf{q}, 0 ; \kappa)=-\frac{\int \mathbf{k} \cdot \mathbf{D}_{11} \cdot \overline{\mathbf{k}} e^{-\kappa \mathbf{e}_{3} \cdot \mathbf{x}_{1}^{0}} P_{N}^{0} d \mathbf{x}^{N, 0}}{\int e^{-\kappa \mathbf{e}_{3} \cdot \mathbf{x}_{1}^{0}} P_{N}^{0} d \mathbf{x}^{N, 0}},
$$

where we have substituted the diffusivity for the mobility: $\mathbf{D}=k T \mathbf{M}$. The denominator of Eq. (31) does not contain any factors proportional to the mean scattering intensity $G$ because we have implicitly taken the $q \rightarrow \infty$ limit (selfdiffusion). By the Riemann-Lebesgue lemma, the Fourier transformation of a square integrable function in the high frequency limit is zero. This is the form of the single particle diffusivity measured in the dilute limit by Holmqvist et al. ${ }^{6}$ via a cumulant expansion. However, our general expression (Eq. (31)) applies for all volume fractions, $\phi$. Equation (31) gives an "evanescent-mean" short-time self-diffusivity that depends on the volume fraction of the particles, the orientation of the scattering wave vector and the penetration depth.

It is more natural to express this mean diffusivity in terms of parallel and perpendicular diffusivities

$$
\begin{aligned}
D_{0}^{S}(\phi, \mathbf{q}, \kappa) & =-\frac{1}{\mathbf{k} \cdot \overline{\mathbf{k}}} \frac{\partial}{\partial t} \log F_{S}(\mathbf{q}, 0 ; \kappa) \\
& =\frac{q_{\|}^{2}\left\langle D_{\|}^{S}\right\rangle+\left(q_{\perp}^{2}+\frac{\kappa^{2}}{4}\right)\left\langle D_{\perp}^{S}\right\rangle}{q_{\|}^{2}+q_{\perp}^{2}+\frac{\kappa^{2}}{4}},
\end{aligned}
$$

where $\mathbf{q} \cdot \mathbf{q}=q_{\|}^{2}+q_{\perp}^{2}, q_{\perp}=\mathbf{q} \cdot \mathbf{e}_{3}$,

$$
D_{\|}^{S}(\phi, \kappa)=\frac{\int \mathbf{D}_{11}:\left(\mathbf{I}-\mathbf{e}_{3} \mathbf{e}_{3}\right) e^{-\kappa \mathbf{e}_{3} \cdot \mathbf{x}_{1}^{0}} P_{N}^{0} d \mathbf{x}^{N, 0}}{\int e^{-\kappa \mathbf{e}_{3} \cdot \mathbf{x}_{1}^{0}} P_{N}^{0} d \mathbf{x}^{N, 0}}
$$


and

$$
D_{\perp}^{S}(\phi, \kappa)=\frac{\int \mathbf{D}_{11}: \mathbf{e}_{3} \mathbf{e}_{3} e^{-\kappa \mathbf{e}_{3} \cdot \mathbf{x}_{1}^{0}} P_{N}^{0} d \mathbf{x}^{N, 0}}{\int e^{-\kappa \mathbf{e}_{3} \cdot \mathbf{x}_{1}^{0}} P_{N}^{0} d \mathbf{x}^{N, 0}} .
$$

The component of the parallel diffusivity is written in terms of the parallel dyads of $\mathbf{D}_{11}$ (proportional to $\mathbf{I}-\mathbf{e}_{3} \mathbf{e}_{3}$ ), which assumes that the suspension structure and hydrodynamics are transversely isotropic. This result again matches with that of Holmqvist et al., ${ }^{6}$ but the diffusivities parallel and perpendicular to the wall are now averaged over the positions of all the particles and not just the scattering test particle. In the dilute limit, $\phi \rightarrow 0$, the initial probability density $P_{N}^{0}$ is such that the positions of all the particles are completely uncorrelated, subject to the requirement that the particles must reside above the plane wall. We are hardly restricted to this limit however, and the above expression is valid over the entire range of volume fractions. Thus, we can probe not just the single particle diffusivity, but the short-time self-diffusivity of one particle immersed in a sea of other particles at any concentration.

Similarly, the time derivative of the logarithm of the intermediate scattering function becomes

$$
\begin{aligned}
\frac{\partial}{\partial t} & \log F(\mathbf{q}, 0 ; \kappa) \\
= & -\frac{1}{(N-1) ! F(\mathbf{q}, 0 ; \kappa)} \\
& \times \int \mathbf{k} \cdot\left[\mathbf{D}_{11}+(N-1) \mathbf{D}_{21} e^{i \mathbf{k} \cdot\left(\mathbf{x}_{2}^{0}-\mathbf{x}_{1}^{0}\right)}\right] \cdot \overline{\mathbf{k}} e^{-\kappa \mathbf{e}_{3} \cdot \mathbf{x}_{1}^{0}} P_{N}^{0} d \mathbf{x}^{N, 0} .
\end{aligned}
$$

This is a natural description of what is termed the wave vector dependent diffusivity, $D(\phi, \mathbf{q}, \kappa)$, which is a function of the wave vector, the volume fraction and the penetration depth. Again, this differs from conventional light scattering in the sense that this is an "evanescent-mean" diffusivity, which is an exponentially weighted average of the self- and interparticle mobilities of particles at different distances from the wall. We write this diffusivity explicitly as

$$
\begin{aligned}
D(\phi, \mathbf{q}, \kappa) & =-\frac{1}{\mathbf{k} \cdot \overline{\mathbf{k}}} \frac{\partial}{\partial t} \log F(\mathbf{q}, 0 ; \kappa) \\
& =\frac{q_{\|}^{2}\left\langle D_{*}\right\rangle+\left(q_{\perp}^{2}+\frac{\kappa^{2}}{4}\right)\left\langle D_{\perp}\right\rangle}{q_{\|}^{2}+q_{\perp}^{2}+\frac{\kappa^{2}}{4}},
\end{aligned}
$$

where

$$
\begin{aligned}
& D_{*}(\phi, \mathbf{q}, \kappa) \\
& =\frac{1}{(N-1) ! F(\mathbf{q}, 0 ; \kappa)} \int\left[\mathbf{D}_{11}+(N-1) \mathbf{D}_{21} e^{i \mathbf{k} \cdot\left(\mathbf{x}_{2}^{0}-\mathbf{x}_{1}^{0}\right)}\right] \\
& \quad:\left(\mathbf{k} \overline{\mathbf{k}}-\left(q_{\perp}^{2}+\frac{\kappa^{2}}{4}\right) \mathbf{e}_{3} \mathbf{e}_{3}\right) \frac{1}{q_{\|}^{2}} e^{-\kappa \mathbf{e}_{3} \cdot \mathbf{x}_{1}^{0}} P_{N}^{0} d \mathbf{x}^{N, 0}
\end{aligned}
$$

and

$$
\begin{aligned}
& D_{\perp}(\phi, \mathbf{q}, \kappa) \\
& =\frac{1}{(N-1) ! F(\mathbf{q}, 0 ; \kappa)} \int\left[\mathbf{D}_{11}+(N-1) \mathbf{D}_{21} e^{i \mathbf{k} \cdot\left(\mathbf{x}_{2}^{0}-\mathbf{x}_{1}^{0}\right)}\right] \\
& \quad: \mathbf{e}_{3} \mathbf{e}_{3} e^{-\kappa \mathbf{e}_{3} \cdot \mathbf{x}_{1}^{0}} P_{N}^{0} d \mathbf{x}^{N, 0} .
\end{aligned}
$$

In conventional light scattering, the collective diffusivity is recovered in the limit that $\mathbf{q} \rightarrow 0$. Applying the same limit here forces $D_{*}(\phi, \mathbf{q} ; \kappa)$ to zero leaving the near-wall collective diffusivity

$$
\begin{aligned}
D_{0}^{C}(\phi, \kappa)= & \int\left[\mathbf{D}_{11} e^{-\kappa \mathbf{e}_{3} \cdot \mathbf{x}_{1}^{0}}+(N-1) \mathbf{D}_{21} e^{-\frac{\kappa}{2} \mathbf{e}_{3} \cdot\left(\mathbf{x}_{2}^{0}+\mathbf{x}_{1}^{0}\right)}\right] \\
& : \mathbf{e}_{3} \mathbf{e}_{3} P_{N}^{0} d \mathbf{x}^{N, 0} /\left\{\int \left[e^{-\kappa \mathbf{e}_{3} \cdot \mathbf{x}_{1}^{0}}+(N-1)\right.\right. \\
& \left.\times e^{-\frac{\kappa}{2} \mathbf{e}_{3} \cdot\left(\mathbf{x}_{2}^{0}+\mathbf{x}_{1}^{0}\right)}\right] P_{N}^{0} d \mathbf{x}^{N, 0} \\
& -G(0,0 ; \kappa) \bar{G}(0,0 ; \kappa)\},
\end{aligned}
$$

which measures the collective hydrodynamics of a suspension but only in the direction normal to the wall. The evanescent wave is unable to recover information about the parallel wall dynamics in the zero scattering angle limit because the decaying intensity of the scattered wave screens out the correlation of any in-plane density fluctuations. Effectively, the averaged hydrodynamics parallel to the wall are $O(\mathbf{q})$ and small. They make no measurable contribution as they are overwhelmed by perpendicular fluctuations. The collective diffusivity measured by evanescent wave spectroscopy is a sum of the self-diffusivity contribution $\left(\mathbf{D}_{11}\right)$ weighted exponentially by the distance of a single particle from the wall and the inter-particle contribution $\left(\mathbf{D}_{21}\right)$ weighted exponentially by the mean distance of any two particles from the wall. In the limit that $\kappa \rightarrow 0$ we see that indeed, the typical collective diffusivity is recovered.

Equations (33), (34), and (39) are new statistical descriptions of what is measured in dynamic light scattering experiments utilizing evanescent waves. These expressions also have the same form as those introduced by Brady ${ }^{4}$ for conventional dynamic light scattering. Namely, they represent the ratio of a hydrodynamic quantity (a weighted average of $\mathbf{D}$ ) to a thermodynamic quantity (a structure factor). These expressions are valid over the whole range of volume fractions and can be used to interrogate directly the results of EWDLS experiments on bounded particles for near-wall diffusivities as well as the results of computational simulations of systems with analogous particles and geometries. Of course, the ideal system for study would be an infinite suspension above a single solid wall. However, this is impossible to realize both experimentally and computationally. Instead, we simulate particles in a parallel wall channel knowing that in the limit that the separation between the channel walls becomes large this approximates the single wall system.

\section{SIMULATION METHODS}

\section{A. Stokesian dynamics}

In the following, we briefly describe the Stokesian dynamics method and refer the reader to more detailed articles when the computational material is beyond the current purview. For a collection of small colloidal particles of negligible Stokes number $\left(\mathrm{St}=\operatorname{Re} \rho_{p} / \rho_{f}\right)$, where $\operatorname{Re}$ is the Reynolds number, $\rho_{p}$ is the density of the particles, and $\rho_{f}$ is 
the density of the fluid), the sum of the forces on the particles are approximately equal to zero, viz.,

$$
0=\mathbf{F}^{H}+\mathbf{F}^{O},
$$

where $\mathbf{F}^{H}$ is the hydrodynamic force on the particles and $\mathbf{F}^{O}$ is any other force on the particles (e.g., Brownian forces and gravitation). As the hydrodynamic force on the particles is related linearly to the particle velocities, the key element of all low-Reynolds-number hydrodynamic simulations is the calculation of this linear couple-the resistance tensor: $\mathbf{R}_{F U}$, or its inverse, the diffusivity: $\mathbf{D}=k T \mathbf{R}_{F U}^{-1}$. One method of calculating this, the Stokesian dynamics technique, ${ }^{15}$ separates the hydrodynamic interactions into two classes: near-field and far-field. In the near-field, the hydrodynamic forces required for relative motion of particles in the fluid are asymptotically large and therefore, the interactions can be treated as pairwise. In the far-field, however, the hydrodynamic interactions are long-ranged (scaling as $r^{-2}$ in the presence of a macroscopic boundary, where $r$ is the distance between particles). As such, the far-field hydrodynamic interactions are many bodied.

The Stokesian dynamics technique determines two sets of forces due to the near- and far-fields, respectively, subject to the constraint that the resulting rigid-body motion of the particles is consistent with both sets of forces. The details are beyond the purview of this article; however, the work by Sierou and Brady ${ }^{16}$ is comprehensive in its development of the method for unbounded systems. Similarly, we refer the reader to the article by Swan and Brady ${ }^{18}$ and the dissertation ${ }^{17}$ which describe in detail the modeling of the hydrodynamic interactions among many particles in a suspension between macroscopic boundaries. Additionally, it describes an accelerated Stokesian dynamics technique for computing these interactions with $O(N \log N)$ computational operations. This is quite rapid given the long-ranged nature of hydrodynamic interactions.

With this approach, we are free to explore the diffusive motion of particles confined between parallel walls in the short-time limit (or at any time via dynamic simulations). We intend to use EWDLS to measure the dynamics of the suspension between the walls, and the full range of penetration depths may be explored. However, for practical reasons it makes sense to only consider penetration depths smaller than $H / 2$, where $H$ is the width of the channel so that we probe the dynamics near the walls and over half the channel. This provides an opportunity to actually multiplex the data by considering the hypothetical situation, where evanescent waves originate from both the top and bottom walls of the channel.

\section{B. Measurement techniques}

Using the simulation techniques described in the previous subsection, we seek to determine the evanescent shorttime self- and collective diffusivities of a dispersion bound between a pair of walls. This can be accomplished using both static and dynamic measurements. We describe in Sec. III B 2 the dynamic measurement technique though we do not employ it here as it measures the same quantities but is more computationally intensive than the static approach.

\section{Static measurements}

A static measurement of the evanescent short-time diffusivity is made by considering the averages in Eqs. (33), (34), and (39) as ensemble averages of the particle velocities due to forces proportional to the evanescent exponential in each respective case. For instance, $D_{\|}^{S}(\phi, \kappa)$ is the ensemble average of the velocity of one particle parallel to the wall due to a force on that particle only, in that same direction and of magnitude $\exp (-\kappa z) /\langle\exp (-\kappa z)\rangle$, where $z$ is the height of the particle above the wall, viz.,

$$
D_{\|}^{S}(\phi, \kappa)=k T \mathbf{e}_{1} \cdot\left\langle\mathbf{M}_{11} \cdot \mathbf{e}_{1} \frac{e^{-\kappa z}}{\left\langle e^{-\kappa z}\right\rangle}\right\rangle .
$$

A similar physical interpretation can be developed for $D_{\perp}^{S}(\phi, \kappa)$, such that

$$
D_{\perp}^{S}(\phi, \kappa)=k T \mathbf{e}_{3} \cdot\left\langle\mathbf{M}_{11} \cdot \mathbf{e}_{3} \frac{e^{-\kappa z}}{\left\langle e^{-\kappa z}\right\rangle}\right\rangle .
$$

The products $\mathbf{M}_{11} \cdot \mathbf{e}_{1} \exp (-\kappa z)$ and $\mathbf{M}_{11} \cdot \mathbf{e}_{3} \exp (-\kappa z)$ are easy to simulate using the methods we described above. One simply proposes that a particle in a particular configuration is forced either parallel or perpendicular to the wall with the appropriate magnitude and then measures the velocity of that particle in the same direction. This calculation is repeated for all the particles in the configuration and the results are averaged. Multiple configurations are generated since the simulations are finite in size, and the same results for all configurations are combined to form the ensemble average. The particles are never moved: however, as the ensemble average is calculated in this strictly static manner.

This approach requires $N$ inversions of $\mathbf{R}_{F U}$ per configuration and is quite slow. However, we can multiply the data recovered from a single inversion by appealing to a stochastic technique similar to the one developed by Sierou and Brady. ${ }^{16}$ Let $\xi_{i}$ be a random, $N$-dimensional vector such that $\left\langle\xi_{i} \xi_{j}\right\rangle=\delta_{i j}$ and apply a force to each particle such that particle $i$ is forced with magnitude $\xi_{i} \exp \left(-\kappa z_{i} / 2\right)$ in the $\mathbf{e}_{1}$ direction. We denote this force on the particles as $\Xi_{S}$ and recognize that the parallel short-time self-diffusivity is simply

$$
D_{\|}^{S}(\phi, \kappa)=k T \frac{\left\langle\Xi_{S} \cdot \mathbf{R}_{F U}^{-1} \cdot \Xi_{S}\right\rangle}{\left\langle\Xi_{S} \cdot \Xi_{S}\right\rangle},
$$

where the ensemble average is now over configurations and instantiations of the randomly distributed forces. A similar expression for $D_{\perp}^{S}(\phi, \kappa)$ can be developed if all the forces are in the $\mathbf{e}_{3}$ direction.

By analogy with the previous expression, consider what happens when the forces on the particles have deterministic magnitude $\exp \left(-\kappa z_{i} / 2\right)$. If the forces all point in the $\mathbf{e}_{3}$ direction, then the short-time collective diffusivity may be written in exactly the same manner, viz.,

$$
D_{0}^{C}(\phi, \kappa)=k T \frac{\left\langle\Xi_{C} \cdot \mathbf{R}_{F U}^{-1} \cdot \Xi_{C}\right\rangle}{\left\langle\Xi_{C} \cdot \Xi_{C}\right\rangle-\left\langle\Xi_{C}\right\rangle \cdot\left\langle\Xi_{C}\right\rangle},
$$

where $\Xi_{C}$ is the collection of the forces on all the particles. These results demonstrate how flexible the light scattering approach to colloid dynamics can be. The evanescent wave samples the suspension as though it were a series of forces 
proportional to $\Xi_{S, C}$ and of precisely the right magnitude to yield these particular self- and collective motions of particles. This physical interpretation can be applied to DLS as well, but it is surprising that it translates so directly to EWDLS. Interestingly, the radiation pressure exerted by the scattered light is proportional to $\mathbf{q} \exp (i \mathbf{k} \cdot \mathbf{x})$. Therefore, while this approach has decomposed the directionality of the scattering, it can be interpreted as measuring the response of the particle to the forcing from radiation pressure. While the scattering may be isotropic so that no net force is imposed on the particle, from a ray-optic perspective, there is indeed a force and the correlated response to that force is the diffusivity. In that way, light-scattering may be thought of as an experimental assertion of the fluctuation-dissipation theorem and realization of the linear response regime.

\section{Dynamic measurements}

Given that the Brownian trajectories of the particles may be computed via dynamic simulation, we seek to measure the intermediate scattering function for a given penetration depth and through that, the evanescent diffusivities. To that end, we are free to choose an evanescent wave vector that will suit our purposes, and because the intermediate scattering function at short times is a convolution of the parallel and perpendicular diffusivities, we must select two independent wave vectors. Since the collective diffusivity comes from the $\mathbf{q} \rightarrow 0$ limit of the scattering function, we choose $\mathbf{q}=0$. This also allows for the direct computation of $D_{\perp}^{S}(\phi, \kappa)$. To measure $D_{\|}^{S}(\phi, \kappa)$, any other non-zero value of $q_{\|}$will suffice. Therefore, we choose $q_{\|}=1$ and $q_{\perp}=0$. With these particular values for the wave vector, one may perform dynamic simulations or experiments and compute the following averages using the known trajectories of particles $\alpha$ and $\beta, \mathbf{x}_{\alpha}^{t}$ and $\mathbf{x}_{\beta}^{t}$, respectively,

$$
\begin{gathered}
F_{S}(0, t ; \kappa)=\left\langle\sum_{\alpha=1}^{N} e^{-\frac{\kappa}{2} \mathbf{e}_{3} \cdot\left[\mathbf{x}_{\alpha}^{t}+\mathbf{x}_{\alpha}^{0}\right]}\right\rangle, \\
F_{S}\left(\mathbf{e}_{1}, t ; \kappa\right)=\left\langle\sum_{\alpha=1}^{N} e^{i \mathbf{e}_{1} \cdot\left[\mathbf{x}_{\alpha}^{t}-\mathbf{x}_{\alpha}^{0}\right]-\frac{\kappa}{2} \mathbf{e}_{3} \cdot\left[\mathbf{x}_{\alpha}^{t}+\mathbf{x}_{\alpha}^{0}\right]}\right\rangle,
\end{gathered}
$$

and

$$
F(0, t ; \kappa)=\left\langle\sum_{\alpha, \beta=1}^{N} e^{-\frac{\kappa}{2} \mathbf{e}_{3} \cdot\left[\mathbf{x}_{\alpha}^{t}+\mathbf{x}_{\beta}^{0}\right]}\right\rangle-\left\langle\sum_{\alpha=1}^{N} e^{-\frac{\kappa}{2} \mathbf{e}_{3} \cdot \mathbf{x}_{\alpha}^{0}}\right\rangle^{2} .
$$

Clearly the time derivative of the logarithm of these functions at $t=0$ are the evanescent diffusivities (perpendicular, Eq. (45); parallel plus the perpendicular component which is independently determined, Eq. (46) and collective, Eq. (47)). For conventional light scattering, the same procedures apply, though only one scattering wave vector is needed since the hydrodynamics and structure of a bulk suspension with volume fraction less than 0.494 are isotropic. For unbounded systems, the long-ranged hydrodynamic interactions are not convergent $\left(\mathbf{D}_{12} \sim r^{-1}\right)$, so that the collective diffusivity $(\mathbf{q}=0)$ cannot be determined directly. Rather, the zero-wave-vectorlimit is extrapolated from the wave vector dependent diffu- sivity for several small, non-zero values of the wave vector. ${ }^{20}$ In the bounded geometry, the hydrodynamic interactions are convergent $\left(\mathbf{e}_{3} \cdot \mathbf{D}_{12} \cdot \mathbf{e}_{3} \sim r^{-3}\right)$, so that no extrapolation is necessary. The collective diffusivity is directly measurable.

\section{RESULTS}

Simulations for suspensions of volume fractions between $10 \%$ and $40 \%$ were performed in a channel of 12 particle radii in width. Equilibrium, hard-sphere configurations of particles were generated via a Monte Carlo method for volume fractions up to $30 \%$ and via a molecular dynamics algorithm for more dense systems. ${ }^{21}$ In the region near the walls, the dynamics of a suspension are largely governed by lubrication interactions corresponding to the drag induced by the wall itself. These hinder the suspension asymptotically such that the self-diffusivity of a particle near a wall scales at its slowest such as $z-a$ (the nearest distance between the particle's surface and the wall). In this range, there is a weak dependence of the hydrodynamics on the channel width. In the results following, the penetration depth is such that the bulk of the suspension sampled is in this near-wall region (i.e., $\kappa H / 2>1$ ). Therefore, the effect of the channel width on the hydrodynamics is minimal (no more than $10 \%$ as determined by comparison with results from larger channels, not shown). Similarly, the near-wall structure has a weak dependence on the channel width for the volume fractions studied. We avoided volume fractions larger than $40 \%$ because in unbounded colloidal dispersions there is a phase boundary at which the suspension would crystallize were it only $10 \%$ more concentrated. The confinement introduces ordering which may propagate via this same mechanism throughout the channel. While the theory and simulations may be used for any channel width and volume fraction, those chosen for this study are appropriate as a model of the single wall system.

The diffusivity computed via simulation of periodic suspensions (bounded or unbounded) has a strong dependence on the size of the periodic simulation cell. It was observed that in channels an aspect ratio of at least two-to-one periodic length to channel width is necessary to get within $5 \%$ of the diffusivity in an aperiodic suspension. ${ }^{18}$ As such, the number of particles simulated (between 300 and 800) is such that the simulation cell always exceeds this aspect ratio. For reference, at least 200 realizations of the equilibrium suspension configuration were used in computing the hydrodynamic contribution to the self- and collective diffusivities. Meanwhile, the structure factors were calculated using more than 3000 realizations of the equilibrium microstructure. The $95 \%$ confidence interval associated with these samples was always smaller than $8 \%$ of the measured value. Error bars are omitted from the figures for clarity, though a confidence interval of $8 \%$ defines a narrow envelope around each data set.

In the limit of large scattering wave vector, the initial slope of the intermediate scattering function is the short-time self-diffusivity. In Fig. 2, this is plotted for various values of penetration depth and volume fraction. Because of the nearby wall, the diffusivity is anisotropic, and the components parallel and perpendicular to the channel wall are distinct. In this case, and for all volume fractions, the near-wall region is 


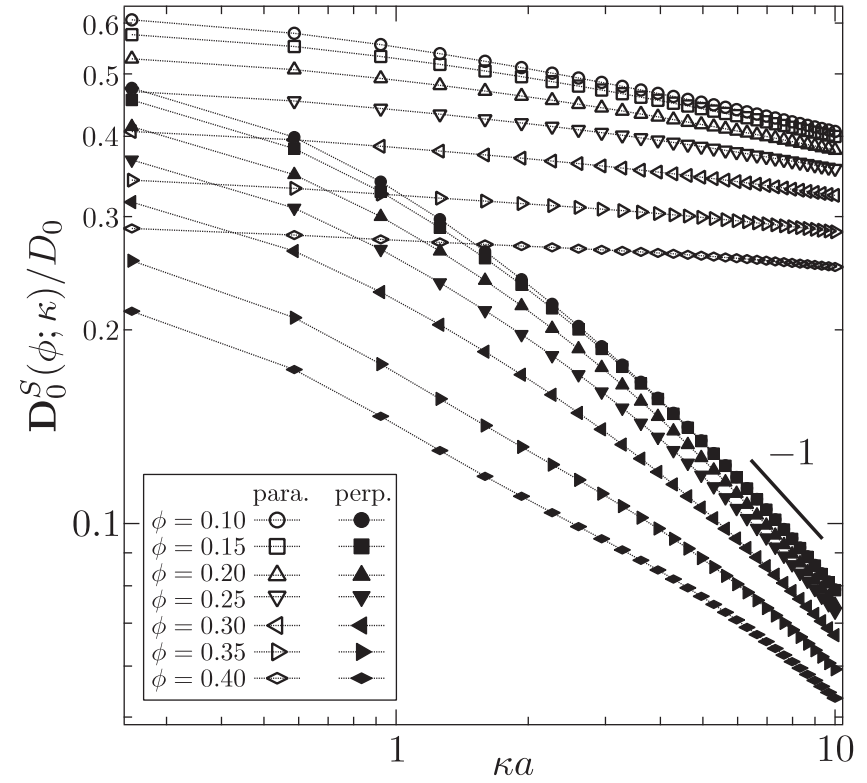

FIG. 2. The evanescent short-time self-diffusivity parallel and normal to the bounding wall plotted as a function of the penetration depth and bulk suspension volume fraction. These are the result of simulations in a channel which is 12 radii in width. It has been shown that the effect of a finite channel width on both the structure and the self-diffusivity is minimal for this range of volume fractions [see, e.g., Ref. 18]. The error is estimated to be less than $8 \%$ of the reported value.

dominated by the lubrication interactions between the particle and the walls. Therefore, the expectation is that the shorttime self diffusivity will scale as $\log (\kappa a)^{-1}$ and $(\kappa a)^{-1}$ in the limit that $\kappa a \rightarrow \infty$, for the parallel and perpendicular components, respectively. This holds for all volume fractions studied though the coefficient of proportionality varies as the particles surrounding the near-wall region, though not directly probed, act as an effective, extra viscous medium.

The self-diffusivity can be computed via direct simulation or observed via optical microscopy as a function of the distance from a wall by measuring the mean force required to move any particle in the suspension of a short distance. The ratio of the particle velocity to the force applied is the short-time self-diffusivity which must be averaged conditionally with respect to that particle's position in the channel and is denoted $\overline{\mathbf{D}}_{0}^{S}(\phi, z)$. The evanescent diffusivity is then

$$
\mathbf{D}_{0}^{S}(\phi, \kappa)=\int \overline{\mathbf{D}}_{0}^{S}(\phi, z) \exp (-\kappa z) n(z) d z / \int \exp (-\kappa z) n(z) d z
$$

such that this transformation allows for the direct comparison of short-time self-diffusivities measured via evanescent wave dynamic light scattering and optical microscopy. Note that extremes in the number density have a deceiving effect on the evanescent wave short-time self-diffusivity as it is in the numerator and denominator of the above expression and would appear to be irrelevant. Instead, extremum in the density (for instance at higher volume fractions) heavily weight the diffusivity with respect to the layering of particles known to occur near a wall. That is, the diffusivity is sampled preferentially at positions very near contact with the wall, in the region where the second layer of particles forms, etc. The extremum retard the self-diffusion at high volume fractions because of the larger numbers of particles in the near-wall region.

It is important to note that the conventional means of determining the short-time self-diffusivity via dynamic light scattering: evaluating the wave vector dependent diffusivity at $q a \sim 4$ for hard-spheres, may not apply. ${ }^{22}$ In particular, the perpendicular component of the short-time self-diffusivity is determined by setting, $q_{\|}=0$. The static structure factor in this case, $F_{S}(\mathbf{q}, 0 ; \kappa)$, is

$$
\langle\exp (-\kappa z)\rangle-\left\langle\exp \left[-\left(i q_{\perp}+\kappa / 2\right) z\right]\right\rangle^{2} .
$$

In the limit of large $q_{\perp}$, the contribution due to the mean scattering intensity (second term in Eq. (49)) tends to zero so that the structure factor becomes $\langle\exp (-\kappa z)\rangle$ (the limiting value desired for computing the self-diffusivity, Eq. (34)). However, the contribution due to the square of the mean scattering intensity decays as $\left(q_{\perp}^{2}+\kappa^{2} / 4\right)^{-1}$. This slow decay may be undesirable experimentally where the maximum scattering angle is limited by practical considerations. The conventional, $q_{\perp} a=4$, may not be large enough for the structure factor to reach its limiting value.

Introducing a small but finite $q_{\|}$reduces the value of the mean scattering intensity. This additional parallel component to the scattering wave vector acts as a Fourier transformation of the particle number density transverse to the boundary. If the suspension is transversely isotropic, there are no spatial variations in the number density in the parallel direction so that this transformation is zero. This eliminates any contribution to the static structure factor belonging to the mean scattering intensity. There are two limitations that bound the value of $q_{\|}$, such that an accurate measurement of the perpendicular self-diffusivity can be made. First, in the limit that $q_{\|} \ll q_{\perp}$ the hydrodynamic contribution to the diffusivity is dominated by the perpendicular component. Second, in order for the mean scattering intensity to go to zero, $q_{\|} L$ must be at least of order unity, where $L$ is the lateral extent of the scattering volume. That is, the scattered wave must fluctuate several times across the scattering volume to give the zero average, we anticipate from a Fourier transformation of the transversely isotropic number density. If the wave oscillates less than once transversely across the volume, then there will be a finite contribution due to the mean volume fraction. This is exactly what happens when $q_{\|}$is identically zero. A more detailed study is required to determine if the heuristic, $q a \sim 4$, for finding the self-diffusivity from experimental data still holds for EWDLS. Recent simulations and measurements of the wave-vector-dependent, evanescent diffusivity suggest this heuristic does not generalize. ${ }^{14}$

In the limit of small scattering wave-vector, the evanescent short-time collective diffusivity is measured-it is plotted in Fig. 3. The evanescent collective diffusivity is a bit peculiar when compared with that for the bulk as it only has components normal to the nearby wall. The evanescent wave selectively weights the probability distribution along that dimension. The hydrodynamic contribution to the collective diffusivity (the sedimentation rate toward the wall weighted by the evanescent decay, or the EWDLS collective diffusivity multiplied by the static structure factor) decreases with increasing volume fraction for $\kappa a<2.5$, while the opposite 


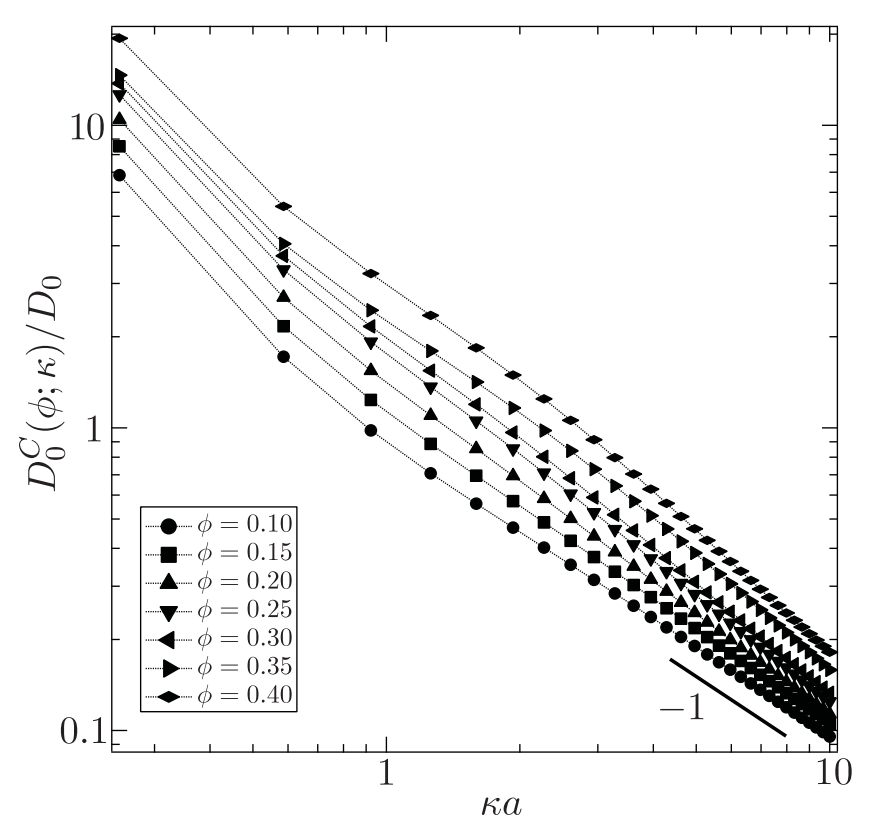

FIG. 3. The evanescent collective self-diffusivity plotted as a function of the penetration depth and bulk suspension volume fraction. These are the result of simulations in a channel which is 12 radii in width. It has been shown that the effect of a finite channel width on both the structure and the sedimentation rate in the channel is minimal for this range of volume fractions [see, e.g., Ref. 18].

trend is observed for smaller penetration depths (not shown). This is contrary to the trend for the sedimentation rate itself, which decreases uniformly with increasing volume fraction. Therefore, structure plays an important role even in the hydrodynamic contribution to the collective diffusivity.

The self-contribution $\left(\mathbf{D}_{11}\right)$ to the collective diffusivity is weighted by the factor $\exp \left(-\kappa z_{1}\right)$ while the pair contribution $\left(\mathbf{D}_{12}\right)$ is weighted by $\exp \left[-\kappa\left(z_{1}+z_{2}\right) / 2\right]$. In the limit of small penetration depths $(\kappa a \rightarrow \infty)$, the ratio of the latter to the former weight is exponentially small for all particle pairs but those with $z_{1} \approx z_{2}$ and $z_{1} \sim a$ (i.e., pairs in which both particles nearly touch the wall). The hydrodynamic interaction between these particular pairs is vanishingly small because of the hydrodynamic screening due to the no-slip condition on the wall. Consequently, in this limit, only $\mathbf{D}_{11}$ contributes to the collective diffusivity, and the evanescent collective diffusivity is proportional to the evanescent self-diffusivity (i.e., $\kappa^{-1}$ ).

The static structure factor in the small wave vector limit is a measure of the number density fluctuations within the scattering volume. Consider a suspension in which the particles are subject to the potential $V(z)=-\kappa z / 2$ in addition to a hard-sphere potential. This is equivalent to a suspension resting in a gravitational potential. From equilibrium thermodynamics, the static structure factor in the small wave-vector limit, $F(0,0 ; \kappa)$ or $S(0 ; \kappa)$, is equivalent to the number density fluctuations in the settled suspension. The structure factor for the settled suspension and the structure factor from EWDLS are the same, and the thermodynamic properties of the settled suspension may be used to infer EWDLS behavior. The grand canonical partition function can be used to show that

$$
F(0,0 ; \kappa) \sim k T \bar{\phi} \bar{\chi}_{T},
$$

where $\bar{\phi}$ and $\bar{\chi}_{T}$ are the mean volume fraction and mean isothermal compressibility of the settled suspension, respectively. ${ }^{1}$ In the limit that $\kappa a \rightarrow 0$, this quantity is $O(1)$ for $\bar{\phi} \ll 1$. If we approximate the isothermal compressibility with the Carnahan-Starling equation of state (the first order contribution for large penetration depths/weak gravitational force), then the static structure factor is proportional to

$$
\frac{(1-\bar{\phi})^{4}}{1+4 \bar{\phi}-2 \bar{\phi}^{2}+\bar{\phi}^{3}} .
$$

The magnitude of the collective diffusivity is set by the inverse of this quantity in the large penetration depth limit. As concentrated suspensions are only weakly compressible, the static structure factor is typically small. Hence, the large value of the collective diffusivity. Conversely, imposing a concentration gradient in the direction normal to the wall yields a large flux of particles because at and near the wall the suspension is effectively incompressible (the wall being unable to deform or move). This drives the particle flux to a degree inversely proportional to the local isothermal compressibility.

Consider a suspension of neutrally buoyant, index of refraction matched colloidal particles seeded dilutely with heavy and optically active particles. The distribution of particles in the suspension, denoted $\tilde{P}_{N}^{0}$ will be Boltzmann such that

$$
\tilde{P}_{N}^{0}=\exp \left[-\left(\frac{4}{3} \pi a^{3}\right)\left(\frac{\Delta \rho g}{k T}\right) \sum_{h} \mathbf{e}_{3} \cdot \mathbf{x}_{h}^{0}\right] P_{N}^{0},
$$

where $4 / 3 \pi a^{3} \Delta \rho g$ is the buoyant force on the heavy particles and the summation over $h$ denotes the heavy particles only. Interestingly, this pre-factor weighting the equilibrium distribution for neutrally buoyant particles, $P_{N}^{0}$, introduces an effective penetration depth $k T /\left(4 / 3 \pi a^{3} \Delta \rho g\right)$. In this way, evanescent-wave-light-scattering-type measurements can be made via a conventional light scattering apparatus. The effect of buoyancy on the dilute, heavy particles is to weight the equilibrium probability distribution with respect to the distance from the boundary where the suspension settles. A scattering volume near that boundary will probe this weighted equilibrium distribution without altering the hydrodynamics. The short-time self-diffusivity of the heavy particles observed via optical microscopy in this suspension and averaged uniformly across the imaged frame measures this same quantity.

A typical penetration depth arising from the buoyancy imbalance for one micron particles with a density difference of $193 \mathrm{~kg} / \mathrm{m}^{3}$ (PMMA in water) at room temperature is $519 \mathrm{~nm}$, which is of the same magnitude as those accessed in evanescent light scattering experiments. $6,12,14$ This value is set experimentally by the low angle of incidence of the internally reflected wave. In the buoyant configuration, $\kappa$ scales as the cube of the particle radius, so that increasing the particle size shifts the diffusivity curves presented in this paper (with respect to the dimensionless independent variable $\kappa a$ ) as $a^{4}$. This is a high degree of sensitivity and can allow easy access to the entire range of penetration depths. One caveat is necessary, however. This method of modeling the evanescent wave light scattering experiment is applicable for comparison of the self-diffusivity only. The reason being that the 
collective diffusivity weights the self-component of the mobility by an exponential decay with respect to the distance of a single particle from the wall, while the pair component is weighted by the average distance of those two particles from the wall. The buoyant distribution does not capture the appropriate weighting for the pair contribution.

The long-time self-diffusion is measured in the limit that the scattering wave vector is small and the correlation time is long. In the limit that the penetration depth approaches infinity, this is simply the bulk long-time self-diffusivity. However, for finite penetration depths, the scattering signal decorrelates rapidly as the fraction of time a particle spends within the scattering volume is small. The measurability of long-time diffusivity is then dictated by the factor $(\kappa H)^{-1}$, where $H$ is the channel width. This reflects the fraction of time the particle spends in the scattering volume and sets the magnitude of the correlated intensity at long times. For $\kappa H \gg 1$, the correlation of the scattering intensity is exponentially small and may be difficult to resolve experimentally. This is an important consideration when studying the long-time dynamics near a boundary.

\section{v. CONCLUSIONS}

Propagating evanescent waves through a dispersion of optically active colloidal particles probes the particle dynamics much as expected from conventional light scattering. As such, it is not so surprising that analysis of the intensity correlation in terms of the intermediate scattering function can by mapped directly on the classical interpretation ${ }^{3}$ by recognizing that the evanescent scattering wave vector is complex (the imaginary component being linearly proportional to the penetration depth of the wave). Performing an analysis which respects the algebraic characteristics of the scattering correlation produces a startlingly simple and powerful result. The time rate of change of the intermediate scattering function is a diffusivity that depends on the scattering wave vector in exactly the same way as in conventional light scattering, with the caveat that the conjugate of the complex wave vector is needed in some places. In the limits of large and small scattering angle, respectively, this diffusivity measures the average short-time self- and collective diffusivities where the average is weighted to decay exponentially with the distance of the scattering particles from the boundary emitting the evanescent wave. This explicit dependence on position is key as it allows the measurement of both the inhomogenous and the anisotropic nature of the hydrodynamic interactions among particles in the presence of a macroscopic boundary. The former task is affected by the penetration depth of the evanescent wave which itself is controlled by the angle of incidence between the scattered wave and the boundary. The latter measurement is affected by the scattering angle itself and in principle the two can be controlled independently. However, this can prove difficult to accomplish with some experimental apparatuses. Fortunately, no such limitation exists computationally and in this article we have determined the self- and collective diffusivities for a variety of penetration depths and volume fractions. In the limit of small penetration depths, the lubrication interactions with the wall dominate both the self- and collective diffusivities while for larger penetration depths, the bulk properties of the suspension can be recovered. The relative influence of interparticle hydrodynamic interactions, hydrodynamic interactions with the boundaries and the influence of the suspension's structure are not easily separable, however. The work presented here provides the necessary foundation for interpreting experimental data and the first predictions of the behavior for hard-sphere colloidal suspensions near a rigid boundary.

${ }^{1}$ B. J. Berne and R. Pecora, Dynamic Light Scattering: With Applications to Chemisty, Biology and Physics (Dover, Mineola, 2003).

${ }^{2}$ W. B. Russel, D. A. Saville, and W. R. Schowalter, Colloidal Dispersions (Cambridge University Press, Cambridge, England, 1989).

${ }^{3}$ J. M. Rallison and E. J. Hinch, J. Fluid Mech. 167, 131 (1986).

${ }^{4}$ J. F. Brady, J. Fluid Mech. 272, 109 (1994).

${ }^{5}$ H. Chew, D. S. Wang, and M. Kerker, Appl. Opt. 18(15), 2679 (1979).

${ }^{6}$ P. Holmqvist, J. K. G. Dhont, and P. R. Lang, Phys. Rev. E 74(2), 021402 (2006).

${ }^{7}$ H. Faxen, Ark. Mat., Astron. Fys. 17(27), (1923); Ph.D. dissertation, Uppsala University, 1921.

${ }^{8}$ C. W. Oseen, "Neuere Methoden und Ergebnisse in der Hydrodynamik," Ph.D. dissertation, Akademische Verlagsgesellschaft, Leipzig, 1928.

${ }^{9}$ J. R. Blake, Proc. Cambridge Philos. Soc. 70, 303 (1971).

${ }^{10} \mathrm{~J}$. Happel and H. Brenner, Low Reynolds Number Hydrodynamics, 2nd ed. (Prentice Hall, Englewood Cliffs, NJ, 1986).

${ }^{11}$ J. W. Swan and J. F. Brady, Phys. Fluids 19(11), 113306 (2007).

${ }^{12}$ V. N. Michailidou, G. Petekidis, J. W. Swan, and J. F. Brady, Phys. Rev. Lett. 102, 068302 (2009).

${ }^{13}$ B. Cichocki, E. Wajnryb, J. Blawzdziewicz, J. K. Dhont, and P. R. Lang, J. Chem. Phys. 132(7), 074704 (2010).

${ }^{14}$ V. N. Michailidou, G. Petekidis, J. W. Swan, and J. F. Brady, "Evanescent wave dynamic light scattering in concentrated colloidal dispersions," Physical Review E, 2011 (in preparation).

${ }^{15}$ L. J. Durlofsky, J. F. Brady, and G. Bossis, J. Fluid Mech. 180, 21 (1987).

${ }^{16}$ A. Sierou and J. F. Brady, J. Fluid Mech. 448, 115 (2001).

${ }^{17} \mathrm{~J}$. W. Swan, "Colloids in confined geometries: hydrodynamics, simulation and rheology," Ph.D. dissertation (California Institute of Technology, May 2010).

${ }^{18}$ J. W. Swan and J. F. Brady, "The hydrodynamics of concentrated and confined colloidal dispersions," Journal of Fluid Mechanics, 2011 (submitted).

${ }^{19}$ J. F. Morris and J. F. Brady, J. Fluid Mech. 312, 223 (1996).

${ }^{20}$ A. M. Leshansky and J. F. Brady, J. Fluid Mech. 527, 141 (2005).

${ }^{21}$ A. Donev, F. H. Stillinger, and S. Torquato, J. Comp. Physics 202(2), 737 (2005).

${ }^{22}$ A. J. Banchio and G. Nägele, J. Chem. Phys. 128, 104903 (2008). 